УДК $538.958 ; 535.016$

DOI: $10.17308 / \mathrm{kcmf} .2019 .21 / 1154$

Поступила в редакцию 18.07.2019

Подписана в печать 15.08.2019

\title{
ТМ ПОЛЯРИЗОВАННЫЕ ПОВЕРХНОСТНЫЕ ВОЛНЫ, РАСПРОСТРАНЯЮЩИЕСЯ ВДОЛЬ ПОВЕРХНОСТИ ФОТОРЕФРАКТИВНОГО КРИСТАЛЛА С НЕЛИНЕЙНЫМ САМОФОКУСИРУЮЩИМ ПОКРЫТИЕМ
}

\author{
(C) 2019 С. Е. Савотченко
}

\author{
Белгородский государственный технологический университет имени В.Г. Шухова \\ ул. Костюкова, 46, 308012, Белгород, Российская Федерация
}

\begin{abstract}
Аннотация. Рассмотрена модель композитной волноводной структуры, основанная на фоторефрактивном кристалле с диффузионным механизмом с нелинейным самофокусирующим покрытием. Показано, что вдоль такой структуры могут распространяться поверхностные волны необыкновенной поляризации. Определены характеристики таких волн. Установлена возможность и условия увеличения или подавления поля на границе фоторефрактивного кристалла.
\end{abstract}

Ключевые слова: поверхностная волна, фоторефрактивный кристалл, константа распространения, композитный волновод, керровская нелинейность.

\section{ВВЕДЕНИЕ}

Особое значение в оптоэлектронике играют различные элементы управления распределением потоками энергии электромагнитных полей оптического диапазона, использующие свойства поверхностных волн (ПВ) и волноводные структуры [1-3]. Физической основой таких элементов, как правило, являются кристаллы, характеризуемые нелинейно-оптическими эффектами, среди которых широкое распространение получили фоторефрактивные кристаллы (ФК) [4]. Теоретическое изучение поверхностных волн в таких кристаллах проводится достаточно давно [5-8]. Тем не менее, возник интерес к изучению локализации поля вдоль контактов кристаллов с различными нелинейно-оптическими эффектами [9-11]. Такого рода контакты могут составлять основу для композитных волноводов, обладающих уникальными свойствами.

В связи с этим в данной работе рассматривается трехслойная структура, представляющая собой ФК, на поверхность которого нанесено покрытие конечной толщины из среды, характеризуемой самофокусирующей нелинейностью. Фоторефрактивная нелинейность в массивном кристалле считается формируемой в результате диффузионного механизма, а в покрытии

$\triangle$ Савотченко Сергей Евгеньевич, e-mail: savotchenkose@mail.ru реализуется эффект Керра, когда коэффициент преломления зависит от квадрата амплитуды напряженности поля (электрического или магнитного). Другая сторона покрытия контактирует со средой, не обладающей никакими нелинейно-оптическими эффектами (диэлектриком, воздухом).

Целью данной работы является изучение особенностей локализации поля вдоль такой композитной структуры, на примере формирования поверхностной волны с ТМ (необыкновенной) поляризацией.

\section{ТЕОРИЯ}

Рассмотрим композитную оптическую структуру, представляющую собой ФК с нанесенным на его поверхность слоем толщины $h$ (покрытия) из нелинейной фокусирующей среды с эффектом Керра, которая контактирует с линейной оптической средой. Границы раздела слоев считаются плоскими и намного тоньше покрытия. Систему координат выберем так, что чтобы граница между нелинейной подложкой и ФК лежала в плоскости $x=0$, а граница раздела между нелинейной подложкой и воздухом (свободная поверхность) лежала в плоскости $x=-h$ перпендикулярно оси $x$. Ось $z$ направлена вдоль слоев. Полярная ось ФК направлена вдоль оси $x$ и ориентирована в плоскости $x y$.

Контент доступен под лицензией Creative Commons Attribution 4.0 License.

The content is available under Creative Commons Attribution 4.0 License. 
Для стационарного распределения поля ТМ-поляризованной ПВ (для которой $E_{y}=0$, $\left.H_{x}=H_{z}=0\right)$, распространяющейся вдоль границ раздела слоев по оси $z$, из системы уравнений Максвелла в рассматриваемом случае получается уравнение для $y$-вой компоненты $H$ вектора напряженности магнитного поля ТМ-волны [8]:

$$
\frac{\partial^{2} H}{\partial x^{2}}+\frac{\partial^{2} H}{\partial z^{2}}+k_{0}^{2} n^{2}(x) H=0
$$

где $k_{0}=2 \pi / \lambda_{0}, \lambda_{0}-$ длина волны света в вакууме, $n(x)$ - пространственная модуляция показателя преломления света в направлении, перпендикулярном границам раздела слоев. Для рассматриваемой слоистой структуры данную зависимость можно представить в виде: $n(x)=n_{L}$ при $x<-h, n(x)=n_{K}+\Delta n_{K}$ при $-h<x<0, n(x)=n_{P}+\Delta n_{P}$ при $x>0, n_{j}$ - невозмущенные показатели преломления (постоянные), $\Delta n_{j}$ - малые нелинейные добавки к ним. Здесь и далее значение индекса $j=P$ соответствует величинам, описывающим свойства $Ф К, j=K-$ величинам, описывающим свойства покрытия, $j=L^{-}$к характеристикам воздуха.

Поскольку рассматривается только скользящее распространение светового пучка, то анизотропией показателя преломления можно пренебречь и использовать одноосное приближение, вследствие чего учитывается изменение показателя преломления только в направлении, перпендикулярном границам раздела слоев.

При диффузионном механизме формирования нелинейности в ФК и пренебрежительно малой темновой интенсивности $I_{d}$ для нелинейной добавки к показателю преломления ФК используют хорошо известное выражение: $\Delta n_{P}(x)=n_{P}^{3} r_{\text {eff }} k_{B} T I^{\prime} / 2 e I[6-8]$, где $I \propto|H|^{2}$, штрихи здесь и далее означают производные по координате $x, r_{\text {eff }}$ - эффективный электрооптический коэффициент, $k_{B}$ - константа Больцмана, $T$ температура, $e$ - модуль заряда электрона. Нелинейная добавка к показателю преломления покрытия с самофокусирующей Керровской нелинейностью пропорциональна интенсивности света: $\Delta n_{K}(x)=\alpha|H|^{2}, \alpha>0$ - коэффициент керровской нелинейности.

Установившееся распределение поля распространяющейся вдоль оси $z$ волны представим в виде: $H(x, z)=H(x) e^{i \beta k_{0} z}$, где $\beta$ - константа распространения. Тогда с учетом $I_{d} \ll I$ и $\Delta n_{P, K} \ll n_{P, K}$, из (1) можно получить уравнения:

$$
H^{\prime \prime}+\left(n_{L}^{2}-\beta^{2}\right) k_{0}^{2} H=0, x<-h,
$$

$$
\begin{gathered}
H^{\prime \prime}+\left(n_{K}^{2}-\beta^{2}\right) k_{0}^{2} H+g|H|^{2} H=0,-h<x<0, \\
H^{\prime \prime}+\mu H^{\prime}+\left(n_{P}^{2}-\beta^{2}\right) k_{0}^{2} H=0, x>0 .
\end{gathered}
$$

где $\mu=2 k_{0}^{2} n_{P}^{4} r_{\text {eff }} k_{B} T / e-$ коэффициент затухания волны в $Ф К, g=2 \alpha k_{0}^{2} n_{K 0}$ - эффективный коэффициент нелинейности в покрытии.

Из условий непрерывности компонент полей на границах раздела слоев вытекают граничные условия:

$$
\begin{gathered}
H(-h-0)=H(-h+0), H(-0)=H(+0), \\
\frac{1}{n_{L}^{2}} H^{\prime}(-h-0)=\frac{1}{n_{K}^{2}} H^{\prime}(-h+0), \\
\frac{1}{n_{K}^{2}} H^{\prime}(-0)=\frac{1}{n_{P}^{2}} H^{\prime}(+0) .
\end{gathered}
$$

Постановка задачи (2)-(6) отличается от рассмотренной в [8] тем, что здесь подложка на поверхности ФК характеризуется нелинейным эффектом Керра с фокусировкой, а в [8] подложка комбинированного волновода представляла собой диэлектрик, не обладающий нелинейно-оптическими эффектами, но с отличающимся от воздуха показателем преломления.

\section{РЕЗУЛЬТАТЫ И ОБСУЖДЕНИЕ}

При $\max \left\{n_{L}, n_{K}, \sqrt{n_{P}^{2}-\mu^{2} / 4 k_{0}^{2}}\right\}<\beta<n_{P}$ и $g>0$ существует ПВ, амплитуда поля в которой убывает без осцилляций в ФК и периодически распределена внутри диэлектрической прослойки, определяемая решениями уравнений (2)-(4), удовлетворяющих граничным условиям (5), (6):

$$
H(x)=\left\{\begin{array}{l}
H_{h} e^{q_{L}(x+h)}, x<-h, \\
k q_{c} \sqrt{\frac{2}{g}} \operatorname{cn}\left(q_{c}\left(x-x_{c}\right), k\right),-h<x<0, \\
A e^{-\mu x / 2} \operatorname{ch}(v x+\delta), x>0,
\end{array}\right.
$$

г д е : $q_{L}^{2}=k_{0}^{2}\left(\beta^{2}-n_{L}^{2}\right), \quad v^{2}=\mu^{2} / 4-k_{0}^{2}\left(n_{P}^{2}-\beta^{2}\right)$, $q_{c}^{2}=k_{0}^{2}\left(\beta^{2}-n_{K}^{2}\right) /\left(2 k^{2}-1\right), k$ - модуль эллиптической функции Якоби, $\mathrm{S}<k^{2}<1$, амплитуды:

$$
\begin{array}{r}
H_{h}=k q_{c} \sqrt{\frac{2}{g}} \operatorname{cn}\left(q_{c}\left(h+x_{c}\right), k\right), \\
A=k q_{c} \sqrt{\left.\frac{2}{g}\left\{v^{2}-\left(\frac{\mu}{2}+\gamma_{c}\right)^{2}\right\} \operatorname{cn}\left(q_{c} x_{c}\right), k\right),} \\
\text { где: } \gamma_{c}=q_{c} \frac{n_{p}^{2}}{n_{K}^{2}} \frac{\operatorname{sn}\left(q_{c} x_{c}, k\right) \operatorname{dn}\left(q_{c} x_{c}, k\right)}{\operatorname{cn}\left(q_{c} x_{c}, k\right)}, \\
\delta=\frac{1}{2} \ln \left(\frac{v+\mu / 2+\gamma_{c}}{v-\mu / 2-\gamma_{c}}\right) .
\end{array}
$$

Параметр $x_{c}$ определяется из уравнения: 


$$
\frac{q_{c}}{q_{L}} \frac{n_{K}^{2}}{n_{L}^{2}}=\frac{\operatorname{sn}\left(q_{c}\left(h+x_{c}\right), k\right) \operatorname{dn}\left(q_{c}\left(h+x_{c}\right), k\right)}{\operatorname{cn}\left(q_{c}\left(h+x_{c}\right), k\right)} .
$$

Период пространственных осцилляций в покрытии $\Lambda_{K}=4 K(k) / q_{c}$, где $K(k)$ - полный эллиптический интеграл первого рода. При удалении от поверхности покрытия поле затухает на расстоянии $l_{L}=1 / q_{L}$. ПВ (7) проникает в ФК на глубину $l_{P}=2 / \mu$ и затухает без осцилляций, но не монотонно в общем случае. Максимум поля в ФК находится на расстоянии

$$
x_{m}=\frac{1}{v}\left\{\operatorname{Arth}\left(\frac{\mu}{2 v}\right)-\delta\right\}
$$

от его границы с покрытием.

ПВ (7) существует при всех допустимых значениях константы распространения из указанного диапазона. Однако при определенных значениях константы распространения возможны специальные режимы распространения ПВ с особенностями распределения профиля поля вдоль слоев.

В частности, если $x_{c}=0$ (или в более общем случае: $\left.q_{c} x_{c}=2 m K(k), m=0,1,2, \ldots\right)$, когда максимум амплитуды поля расположен на границе ФК и покрытия, то выражение (10) можно трактовать как дисперсионное уравнение, определяющее зависимость константы распространения от оптических характеристик структуры. Тогда при $x_{c}=0$ в случае тонкого покрытия в приближении $q_{c} h<<1$ из дисперсионного уравнения (10) можно получить такую зависимость в явном виде:

$$
\beta^{2}=n_{K}^{2}+\beta_{c 0}^{2}\left\{1 \pm\left[1+\left(n_{K}^{2}-n_{L}^{2}\right) / 2 \beta_{c 0}^{2}\right]^{1 / 2}\right\},
$$

где $\beta_{c 0}=\left(2 k^{2}-1\right) n_{K}^{2} / \sqrt{2} h k_{0} n_{L}^{2}$. Если $n_{L}<n_{K}$, то ПВ с константой распространения, определяемой зависимостью (12), существует при любых допустимых значениях оптических характеристик слоев и эллиптического модуля. Если $n_{L}>n_{K}$, то ПВ существует при условии $k^{2}>1 / 2+h k_{0}\left(n_{L}^{2}-n_{K}^{2}\right)^{1 / 2} n_{L}^{2} / n_{K}^{2}$.

Если дополнительно предположить, что коэффициент преломления диэлектрика и невозмущенный коэффициент преломления покрытия практически совпадают $\left(n_{L} \sim n_{K}\right)$, то получается более простая форма зависимости константы распространения:

$$
\beta^{2}=n_{K}^{2}+\left\{\left(2 k^{2}-1\right) / h k_{0}\right\}^{1 / 2} .
$$

Если $q_{c} x_{c}=(2 m+1) K(k)$, то поле на границе между ФК и покрытием обращается в ноль. Из дисперсионного уравнения (10) в этом случае следует, что такая ситуация реализуема только в покрытии конечной толщины, поскольку при данном условии уравнение (10) имеет решение при $2 K(k)<q_{c} h<3 K(k)$, что не возможно для тонкопленочного покрытия.

Отличительной особенностью распространения ПВ вдоль ФК является то, что могут существовать и затухающие с осцилляциями волны. При $\max \left\{n_{L}, n_{K}\right\}<\beta<\sqrt{n_{P}^{2}-\mu^{2} / 4 k_{0}^{2}}$ и $g>0$ существует ПВ второго типа:

$$
H(x)=\left\{\begin{array}{l}
H_{h} e^{q_{L}(x+h)}, x<-h, \\
k q_{c} \sqrt{\frac{2}{g}} \operatorname{cn}\left(q_{c}\left(x-x_{c}\right), k\right),-h<x<0,(14) \\
H_{0} e^{-\mu x / 2} \cos (p x+\varphi) / \cos \varphi, x>0,
\end{array}\right.
$$

где $p^{2}=-v^{2}$, амплитуда поля на границе ФК и покрытия:

$$
H_{0}=\sqrt{\frac{2}{g}} k q_{c} \operatorname{cn}\left(q_{c} x_{c}, k\right)
$$

фаза волны:

$$
\varphi=-\frac{1}{p} \operatorname{arctg}\left(\frac{\mu}{2}+\gamma_{c}\right),
$$

а остальные параметры такой ПВ определяются прежними формулами (такими же, как и для ПВ первого типа). Также как и ПВ первого типа, волна (14) проникает вглубь ФК на расстояние $l_{p}$, но ее амплитуда спадает осциллирующим образом с периодом осцилляций $\Lambda_{p}=2 \pi / p$. Данный период зависит от коэффициента затухания $\mu$, который, в свою очередь, зависит от оптических характеристик ФК и его температуры. Следовательно, регулируя температуру ФК, можно управлять глубиной локализации поля в нем и периодом пространственных осцилляций.

\section{ЗАКЛЮЧЕНИЕ}

В работе показано, что вдоль поверхности ФК с покрытием из нелинейной керровской самофокусирующей среды могут распространяться ПВ с различными формами затухания, определяющими локализацию электромагнитного поля. Определены характеристики таких ПВ. Указаны режимы, при которых поле на границе ФК с покрытием может достигать максимального значения, либо нулевого. Показана возможность управления профилем локализации электромагнитного поля путем изменения температуры композитной структуры.

Результаты данной работы могут быть полезными при разработке различных элементов оптоэлектронных устройств на основе композитных волноводов, работающих при варьируемых температурных режимах. 


\section{КОНФЛИКТ ИНТЕРЕСОВ}

Автор декларируют отсутствие явных и потенциальных конфликтов интересов, связанных с публикацией настоящей статьи.

\section{СПИСОК ЛИТЕРАТУРЫ}

1. Strudley T., Bruck R., Mills B., Muskens O. L. An ultrafast reconfigurable nanophotonic switch using wavefront shaping of light in a nonlinear nanomaterial // Light: Science \& Applications, 2014, v. 3, p. e207. DOI: $10.1038 / \mathrm{lsa} .2014 .88$

2. Naim Ben Ali. Narrow stop band microwave filters by using hybrid generalized quasi-periodic photonic crystals // Chinese J. of Phys., 2017, v. 55, pp. 2384-2392. DOI: 10.1016/j.cjph.2017.10.008

3. Bettella G., Zamboni R., Pozza G., Zaltron A., Montevecchi C., Pierno M., Mistura G., Sada C., Gauthier-Manuel L., Chauvet M. $\mathrm{LiNbO}_{3}$ integrated system for opto-microfluidic sensing // Sensors and Actuators B: Chem., 2019, v. 282, pp. 391-398. DOI: 10.1016/j.snb.2018.10.082.

4. Петров М. П., Степанов С. И., Хоменко А. В. Фоторефрактивные кристаллы в когерентной оптике. СПб.: Наука, С.-Петерб. отд-ние, 1992, 317 с.

5. Белый В. Н., Хило Н. А.Поверхностные световые волны на границе фоторефрактивного кристалла с диффузионно-дрейфовым механизмом нелинейности // Письма в ЖТФ, 1997, т. 23(12), с. 31-36.
6. Шандаров С. М., Шандаров Е. С. Фоторефрактивные щелевые волны // Письма в ЖТФ, 1997, т. 23(15), c. 30-35.

7. Четкин С. А., Ахмеджанов И. М. Оптическая поверхностная волна в кристалле с диффузионной фоторефрактивной нелинейностью // Кв. электр., 2011, т. 41(11), с. 980-985.

8. Усиевич Д. Х., Нурлигареев Б. А., Сычугов В. А., Ивлева Л. И. Комбинированный волновод на фоторефрактивном кристалле // Кв. электр., T. 41(11), c. 924-928.

9. Savotchenko S. E. Nonlinear surface TM waves in a Kerr defocusing nonlinear slab sandwiched between photorefractive crystals // Solid State Communications, 2019. v. 296(7), pp. 32-36. DOI: 10.1016/j. ssc.2019.04.008

10. Савотченко С. Е. Влияние температуры на перераспределение потока энергии, уносимого поверхностными волнами вдоль границы раздела кристаллов с различными механизмами формирования нелинейного отклика // Письма в ЖЭТФ, 2019, т. 109(11), с. 778-782. DOI: 10.1134/ S0370274X19110109

11. Савотченко С. Е. Нелинейные поверхностные волны на границе раздела оптических сред с различными механизмами индуцирования нелинейности // ЖЭТФ, 2019, т. 156(8), с. 195-204. DOI: $10.1134 /$ S0044451019080017

\title{
TM POLARIZED SURFACE WAVES PROPAGATING ALONG THE SURFACE OF A PHOTOREFRACTIVE CRYSTAL WITH NONLINEAR SELF-FOCUSING COATING
}

\author{
(C) 2019 S.E. Savotchenko ${ }^{\bowtie}$ \\ Belgorod State Technological University named after V. G. Shukhov \\ 46, Kostyukovastr., 308012 Belgorod, Russian Federation
}

\begin{abstract}
Objective. A model of a composite waveguide structure based on a photorefractive crystal with a diffusion mechanism with a nonlinear self-focusing coating is considered. The Kerr effect is realized in the coating when the refractive index depends on the square of the amplitude of the field strength (electric or magnetic). The other side of the coating is in contact with a medium that does not have any non-linear optical effects (dielectric or air).

Purpose. The aim of this work is to study the localization features of the field along such a composite structure using, for example, the formation of a surface wave with TM polarization. Methods and methodology. The system of Maxwell equations is used for describing the stationary distribution of the TM-polarized surface wave field propagating along the interfaces
\end{abstract}

$\triangle$ Savotchenko Sergey E., e-mail: savotchenkose@mail.ru 
of the layers. A special form of dependence of the refractive indices of the layers of the composite waveguide is chosen.

Results. A system of nonlinear equations is formulated for a component of the magnetic field strength of a TM-polarized surface wave. The two types of solution of such system are found. The solutions satisfy the conditions at the interfaces between the layers and the disappearance of the field at infinity in the truncated layers direction. Solutions of two types exist in different ranges of propagation constant values. They describe the surface waves with different localization profiles across the layers. The first type of waves attenuates without oscillation and the second one attenuates with oscillations. It is shown that by adjusting the temperature of a photorefractive crystal, one can control the depth of localization of the field in it and the period of spatial oscillations. The possibility of increasing or suppressing the field at the boundary of a photorefractive crystal has been established.

Conclusions. It is shown that the TM-polarized waves can propagate along considered waveguide structure surface. The properties of such waves are determined in dependence of optical characteristics of the waveguide.

Keywords: surface wave, photorefractive crystal, propagation constant, composite waveguide, Kerr nonlinearity.

\section{CONFLICT OF INTEREST}

The authors declare the absence of obvious and potential conflicts of interest related to the publication of this article.

\section{REFERENCES}

1. Strudley T., Bruck R., Mills B., Muskens O. L. An ultrafast reconfigurable nanophotonic switch using wavefront shaping of light in a nonlinear nanomaterial.Light: Science \& Applications, 2014, v. 3, p. e207. DOI: $10.1038 /$ lsa.2014.88.

2. Naim Ben Ali. Narrow stop band microwave filters by using hybrid generalized quasi-periodic photonic crystals.Chinese J. of Phys., 2017, v. 55, pp. 23842392. DOI: DOI: 10.1016/j.cjph.2017.10.008

3. Bettella G., Zamboni R., Pozza G., Zaltron A., Montevecchi C., Pierno M., Mistura G., Sada C., Gauthier-Manuel L., Chauvet M. $\mathrm{LiNbO}_{3}$ integrated system for opto-microfluidic sensing. Sensors and Actuators B: Chem., 2019, v. 282, pp. 391-398. DOI: 10.1016/j.snb.2018.10.082.

4. Petrov M. P., Stepanov S. I., Homenko A. V. Fotorefraktivnyekristally $v$ kogerentnojoptike[Photorefrac tive crystals in coherent optics]. Saint Petersburg, Nauka Publ., 1992, 317 p. (in Russ.)

5. Belyi V. N., Khilo N. A. Surface light waves at the border of a photorefractive crystal with a diffusion- drift nonlinearity mechanism.Tech. Phys. Lett., 1997, v. 23(12), pp. 31-36. (in Russ.)

6. Shandarov S. M., Shandarov E. S. Photorefractive slit waves.Tech. Phys. Lett., 1997, v. 23(15), pp. 30-35. (in Russ.)

7. Chetkin S. A., Akhmedzhanov I. M. Optical surface wave in a crystal with diffusion photorefractive nonlinearity.Quant. Electr., 2011, v. 41(11), pp. 980985. (in Russ.)

8. Usievich D. Kh., Nurligareev B. A., Sychugov V. A., Ivleva L. I. Combined waveguide on a photorefractive crystal.Quant. Electr., v. 41(11), pp. 924-928. (in Russ.)

9. Savotchenko S. E. Nonlinear surface TM waves in a Kerr defocusing nonlinear slab sandwiched between photorefractive crystals. Solid State Communications, 2019. v. 296(7), pp. 32-36. DOI: 10.1016/j. ssc.2019.04.008.

10. Savotchenko S. E. Effect of the Temperature on the Redistribution of an Energy Flux Carried by Surface Waves along the Interface between Crystals with Different Mechanisms of Formation of a Nonlinear Response. JETP Lett., 2019, v. 109(11), pp. 744-748. DOI: 10.1134/S0021364019110146

11. Savotchenko S. E. Nonlinear surface waves at the interface of optical media with various mechanisms for inducing nonlinearity. JETP, 2019, v. 129(2), pp. 159-167. DOI: 10.1134/S1063776119070100.
Савотченко Сергей Евгеньевич - д. ф. - м. н, доцент, профессор кафедры высшей математики, Белгородский государственный технологический университет имени В. Г. Шухова, Белгород, Российская Федерация; e-mail: savotchenkose@ mail.ru. ORCID iD 0000-0002-7158-9145.
Savotchenko Sergey E. - Dr. Sci. (Phys.-Math.), Associate Professor, Professor of High Mathematics Department, Belgorod State Technological University named after V. G. Shukhov, Belgorod, Russian Federation; e-mail: savotchenkose@mail.ru. ORCID iD 0000-0002-7158-9145. 